\title{
Enseñanza de 'temas controversiales' en la asignatura de historia y ciencias sociales desde la perspectiva de los profesores*
}

\author{
Teaching of 'controversial issues' in history and social science from teachers' perspective
}

\author{
Ensino de 'temas controvertidos' na disciplina de história e ciências sociais \\ na perspectiva dos professores
}

\author{
$M^{a}$ Isabel Toledo Jofré, ${ }^{a}$ Abraham Magendzo Kolstrein, ${ }^{b}$ Virna Gutiérrez Gianella, ${ }^{c}$ \\ Ricardo Iglesias Segura. ${ }^{d}$ \\ a Universidad Diego Portales, Chile. Fono: 56-2-26768637. \\ Correo electrónico: maria.toledo@udp.cl \\ ${ }^{\text {b } U n i v e r s i d a d ~ A c a d e m i a ~ d e ~ H u m a n i s m o ~ C r i s t i a n o, ~ C h i l e . ~ F o n o: ~ 56-2-27878000 . ~}$ \\ Correo electrónico: abrahammagendzo@gmail.com \\ c Universidad Diego Portales, Chile. Fono: 56-2-26768136. \\ Correo electrónico: virna.gutierrez@udp.cl \\ d Pontificia Universidad Católica de Valparaíso, Chile. Fono: 56-32-2274806. \\ Correo electrónico: riglesia@ucv.cl
}

\section{RESUMEN}

Los 'temas controversiales' son tópicos sobre los cuales diferentes grupos han construido argumentaciones irreconciliables. Por ello, dividen a la sociedad. Algunos están presentes en el curso de historia. El objetivo de la investigación es describir la enseñanza de los 'temas controversiales' en los cursos de historia del primer ciclo de enseñanza secundaria en Chile. Se utiliza una muestra no probabilística, aleatoria estratificada con afijación proporcional. Se aplica un cuestionario auto-administrado a 111 profesores de establecimientos municipalizados, particulares subvencionados y particulares pagados. Se realiza un análisis estadístico descriptivo y se construye una tipología de los profesores. Se establece el grado de conflictividad de los temas y su enseñanza, se analiza la posición de los profesores para enseñar los temas y se describen las acciones que realizan en la sala de clases. Los profesores evitan abordar los temas controversiales y se declaran 'neutrales', aunque sus prácticas se distancian de esa posición.

Palabras clave: 'temas controversiales', enseñanza de la historia, enseñanza secundaria.

\begin{abstract}
'Controversial issues' are topics under which different groups have built irreconcilable arguments on. Therefore, they divide society. Some of these topics are now present in the teaching of history. Thus, the purpose of this research is to describe the teaching of 'controversial issues' in history subjects in secondary school. A nonstatistical randomly stratified sample with proportional representation was used. A self-administered questionnaire was applied to 111 teachers of public schools, private subsidy schools and private paid schools. A statistically

Este artículo se ha escrito en el marco del Proyecto FONDECYT 1120742: "Estudio sobre la enseñanza de los 'temas controversiales' en el curso de Historia, Geografía y Ciencias Sociales, durante el primer ciclo de Enseñanza Media. Análisis de las estrategias de afrontamiento: Adoctrinamiento versus Neutralidad", donde también colaboran Bastián Abarca Hoffman, Magíster @ Pensamiento Contemporáneo (UDP), Sebastián García Ralph, Magíster @ en Educación Informática Educativa (UCH), Karla Ulloa Flores, Socióloga (UDP) y Patricio Enrique Saavedra Díaz, Estudiante de Psicología (UDP).
\end{abstract}


descriptive analysis was made and a teachers' typology was made. A degree of debatable nature was established in relation to the 'controversial issues' and their teaching. Teachers' approach to teaching these topics was analyzed and activities in the classroom were described. Teachers avoided tackling controversial topics they considered themselves as being 'neutral' even though their performances were far away from that position.

Key words: 'controversial issues', history teaching, secondary school.

\section{RESUMO}

'Temas controvertidos' são aqueles sobre os quais diferentes grupos têm construído argumentações irreconciliáveis. Por esta razão, dividem a sociedade. Alguns destes estão presentes no curso de história. O objetivo da investigação é descrever o ensino dos 'temas controvertidos' nos cursos de história do primeiro ciclo do ensino secundário no Chile. Utiliza-se uma amostragem não probabilística, aleatória estratificada com afixação proporcional. Aplica-se um questionário autoadministrado a 111 professores de estabelecimentos municipais, particulares subvencionados e particulares pagos. Realiza-se uma análise estatístico-descritiva e constrói-se uma tipologia dos professores. Estabelece-se o grau de embate de ideias dos temas e de seu ensino, analisa-se a posição dos professores para ensinar tais temas e descrevem-se as ações realizadas por eles em sala de aula. Os professores evitam abordar os temas controvertidos e se declaram 'neutros', ainda que suas práticas se distanciem de esta posição.

Palavras chave: 'temas controvertidos', ensino de história, ensino secundário.

\section{INTRODUCCIÓN}

Actualmente, las escuelas están demandadas a formar sujetos reflexivos, con capacidad de deliberación y juicio crítico, competentes para analizar dilemas éticos de alcance social y público, y con habilidad argumentativa para defender sus ideas (Osorio, 2007). Para alcanzar estos propósitos, es necesario que la escuela reconozca la existencia de conflictos sociales y los incorpore al currículum, y que también explicite las tensiones y las diferencias existentes en el ámbito científico de donde surge el conocimiento. Esto, porque la escuela se ha presentado, por lo general, como una institución neutral, ajena a las ideologías y los aconteceres políticos. Los saberes se enseñan como si fueran consensuales, se oculta el desacuerdo y la controversia. Se omiten las diferencias epistemológicas y teóricas y las luchas de poder entre los científicos y entre las instituciones productoras de conocimiento.

El conflicto también es ocultado cuando se analiza la sociedad. La sociedad es presentada como un modelo de cooperación, donde cada elemento cumple una función que contribuye a la mantención de la continuidad social. Ahí, el conflicto social es incompatible e incluso amenazante del orden social, puesto que se concibe como un quebrantamiento a lo establecido (Apple, 2008). La institución escolar ha obrado bajo el supuesto de que, eliminando los conflictos y las controversias, se pueden construir escuelas armoniosas. Por ello, los 'temas controversiales' han quedado fuera de la escuela, pero se ha olvidado que estos tópicos están presentes en la vida de los estudiantes, puesto que ellos son informados por los medios de comunicación (Gerson, 1997) y son parte de su vida cotidiana.

Los 'temas controversiales' son tópicos sobre los cuales no existe un consenso (Crick, 1998 Cit. en Levinson, 2006). Son temas a partir de los cuales se generan conflictos (Vashist, 1993 Cit. en Asimeng-Boahene, 2007). Estos temas dividen a la sociedad porque existen grupos que producen argumentaciones diferentes sobre ellos (Bailey, 1975 Cit. en Oulton, Day, Dillon \& Grace, 2004; Stradling, 1984 Cit. en Harwood \& Hahn, 1990), y/o proponen resolver un problema de manera distinta (Advisory Group On Citizenship, 1998). Estos tópicos provocan divisiones porque los grupos producen explicaciones y/o soluciones que son irreconciliables (Crick, 1998 Cit. en Levinson, 2006; Wales \& Clarke, 2005 Cit. en 
Levinson, 2006; Oulton, Day, Dillon \& Grace, 2004; Stradling, 1985 Cit. en Oulton et al., 2004), y se originan en diferentes sistemas de creencias y valores que sustentan los grupos (Stradling, 1984 Cit. en Harwood \& Hahn, 1990). Las argumentaciones en torno a un 'tema controversial' suelen ser públicas y reconocidas por los grupos en disputa, aunque un grupo puede sentirse limitado en la expresión de sus opiniones (Levinson, 2006). Por lo tanto, pueden desatar emociones y sentimientos entre quienes forman parte de los grupos en conflicto (Vashist, 1993 Cit. en Asimeng-Boahene, 2007).

Ante los 'temas controversiales' los profesores toman diferentes posiciones:

- El profesor 'evitativo' reconoce el contenido como un tema polémico y no lo incluye en sus clases (Vandenberg, 2009; Corngold \& Waddington, 2006), pues considera que abordarlo desataría conflictos en la comunidad escolar (Trilla, 1995).

- El profesor 'objetivo' aborda sólo los aspectos que han sido objetivados, los que no producen polémica (Vandenberg, 2009).

- El profesor 'negador' reconoce la existencia del 'tema controversial' y de sus diversas interpretaciones, pero presenta únicamente su propia perspectiva del tópico (Hess, 2004).

- Un profesor es 'neutral' cuando, ante un 'tema controversial', presenta diferentes perspectivas (Stradling, Noctor \& Baines, 1984 Cit. en Cotton, 2006; Hess, 2004), actúa como moderador de la discusión (Stenhouse, 1983 Cit. en Oulton et al., 2004), y evita utilizar su autoridad para influir a los estudiantes (Schools Council and Nuffield Humanities Project, 1970 Cit. en Cotton, 2006). Por un lado, practica la 'neutralidad activa' si no manifiesta su opinión (Vandenberg, 2009; Joseph, 1988 y Singh, 1988 Cit. en Harwood, 2001; Trilla, 1995) ni apoya ninguna conclusión (Corngold \& Waddington, 2006); por otro lado, practica la 'neutralidad comprometida' cuando a la diversidad de interpretaciones presentadas agrega la suya indicando que se trata de su opinión (Kelly, 1986 Cit. en Oulton et al., 2004), y se manifiesta a favor de una de las conclusiones a las que se llega, pero indica que su conclusión podría ser errónea. Además, promueve la revisión permanente de todas las argumentaciones (Corngold \& Waddington, 2006).

- El profesor 'crítico' presenta las interpretaciones de uno de los grupos en disputa (Hess, 2004): la de los oprimidos (Applebaum, 2003 Cit. en Corngold \& Waddington, 2006). Su propósito es que los estudiantes aprendan a reflexionar y asuman una actitud crítica sobre las relaciones sociales de las cuales forman parte (Marcroft, 1990 Cit. en Corngold \& Waddington, 2006).

- Un profesor es 'adoctrinador' si, después de un proceso de aprendizaje, el estudiante ha interiorizado argumentos que no resisten un análisis racional (Kleinig, 1982 Cit. en Corngold \& Waddington, 2006) ni un análisis crítico (Oulton, Dillon \& Grace, 2004 Cit. en Oulton et al., 2004), y/o cuando los estudiantes aceptan como verdaderas argumentaciones no verificadas (Finkin, Post, Nelson, Benjamin \& Combest, 2007). Se opera un proceso de inculcación (Cuypers \& Ishtiyaque, 2006) en el que se presentan proposiciones dogmáticamente (Finkin et al., 2007). También se adoctrina cuando se expone sólo una versión de la historia (Hocutt, 2005), y cuando no se presentan evidencias (Hanks, 2008; Siegel, 1998 Cit. en Cuypers \& Ishtiyaque, 2006; Hocutt, 2005; Merry, 2005).

Algunos profesores reconocen la contribución de la enseñanza de los 'temas controversiales' para los estudiantes (Barton \& Mc Cully, 2007; Gayford, 2002). Mientras que otros afirman que no deben ser incorporados al currículum, porque generan confusión entre objetivos educacionales y socio-políticos (Wilson, 1986 Cit. en Finn, 1990). Otros 
Estudios Pedagógicos XLI, Nº 1: 275-292, 2015

ENSEÑANZA DE 'TEMAS CONTROVERSIALES' EN LA ASIGNATURA DE HISTORIA Y CIENCIAS SOCIALES

DESDE LA PERSPECTIVA DE LOS PROFESORES

sostienen que no son parte del currículum, o que su trabajo obstaculiza la enseñanza de los temas que la escuela debe cubrir (Van Rooy, 2000).

Muchos profesores cuya ideología u opción política difiere de los postulados oficiales, se sienten inhibidos de expresar sus opiniones. Piensan que al hacerlo pueden ser acusados de enemigos de la sociedad, como sucedió durante la era de McCarthy (Bollinger, 2005 Cit. en Williams, 2006), y durante las dictaduras militares en América Latina (Toledo, Veneros y Magendzo, 2006). Entre los profesores existe temor al conflicto que se puede desatar con las autoridades cuando se manifiestan visiones diferentes de sociedad (Asimeng-Boahene, 2007). También hay quienes piensan que enseñarlos podría molestar a los directivos y/o a otros miembros de la comunidad escolar (Jacobs, 2010), u ocasionar que se les acuse de adoctrinamiento, especialmente, si se cuestiona el modelo económico imperante (Carr, 2011).

Los profesores indican que la complejidad de los temas dificulta su enseñanza (Clarke, 2005). Además, reconocen falta de conocimientos sobre los tópicos (Dawson, 2001) que están desactualizados en sus disciplinas (Van Rooy, 2000), y no conocen material didáctico para trabajarlos (Dawson, 2001). Algunos profesores consideran que es negativo explicitar sus opiniones (Cotton, 2006), o les incomoda discutir temas políticos con los estudiantes (Byford, Lennon \& Russell, 2009). Les preocupa manifestar su opinión en un debate (Cross \& Price, 1996), y consideran que no es adecuado implicarse en las polémicas porque tienen dificultades para mantenerse imparciales. Por ello desean presentar un discurso 'objetivo', aunque es casi imposible asumir una postura neutral (Cotton, 2006). Piensan que podrían inducir a los estudiantes a adoptar posiciones divergentes o contrarias a sus propios planteamientos. Por estas razones, los profesores pueden llegar a auto-censurarse para disminuir posibles reacciones negativas de los pares o de los padres (Asimeng-Boahene, 2007).

Los profesores, además, se preocupan por la gestión de la discusión: de las emociones, de las preguntas de los estudiantes y de su posicionamiento sobre los temas (Levitt \& Longstreet, 1993). Temen perder el control de la discusión (Byford, Lennon \& Russell, 2009; Baker, Jensen \& Kolb, 2002 Cit. en Ezzedeen, 2008) o influir en su contenido (Bridges, 1979). Otros profesores piensan que pueden perder su credibilidad cuando se desatan conflictos de opinión con los estudiantes (Sims, 2004), o creen que podrían producir conflictos y divisiones entre los estudiantes (Jacobs, 2010). También les resulta difícil garantizar la participación de los estudiantes que tienen posturas minoritarias (Cotton, 2006). Según algunos profesores, los estudiantes manifiestan resistencias emocionales a aceptar puntos de vista diferentes a los suyos y a reconocer la existencia de hechos que se contrapongan con sus interpretaciones (Asimeng-Boahene, 2007). Otros piensan que los estudiantes carecen de experiencia para analizar los 'temas controversiales' (Levitt \& Longstreet, 1993), o que no están cognitivamente capacitados para abordarlos (Finn, 1990).

También es necesario considerar que la enseñanza de los 'temas controversiales' es más compleja en las sociedades que han vivenciado guerras, genocidios, conflictos étnicos, religiosos y/o dictaduras que han dividido profundamente la sociedad (Asimeng-Boahene, 2007). En el caso de Chile, sólo basta con ejemplificar que la interpretación del pasado desde el golpe de Estado de 1973 se encuentra polarizada (Moulian, 2010; Huneeus, 2003; Stern, 2000; Gazmuri, s/f). La memoria colectiva aún divide a la sociedad chilena y esto incluye a los estudiantes (Toledo, Veneros y Magendzo, 2006). Se ha establecido que, el año 2007 , el $34,7 \%$ de los profesores de $6^{\circ}$ básico no cubrió la enseñanza del golpe de Estado y el régimen militar (Toledo y Magendzo, 2007), y el año 2005, el 55\% de los profesores de segundo medio tampoco lo enseñó (Toledo, Magendzo y Gazmuri, 2010). 
Además, el 24\% de sus estudiantes considera que los profesores imponen sus ideas sobre la historia reciente (Toledo y Magendzo, 2009).

En este contexto, se realizó una investigación que tuvo por propósito dar cuenta de la enseñanza de los 'temas controversiales' en los cursos de historia durante el primer ciclo de enseñanza media. En primer lugar, se identificaron los 'temas controversiales' presentes en los Programas del Sector de Historia, Geografía y Ciencias Sociales de primer y segundo año medio. Luego, el trabajo se orientó a describir las prácticas pedagógicas de los profesores de historia cuando enseñan 'temas controversiales', y se construyó una tipología de estrategias de posicionamiento frente a su enseñanza. Aunque la investigación también considera el estudio de la percepción que tienen los estudiantes sobre la enseñanza de estos tópicos, en este artículo sólo se reporta lo informado por los profesores.

\section{METODOLOGÍA}

La población corresponde a los establecimientos educacionales urbanos de la Región Metropolitana de Santiago que imparten educación media. El marco muestral es el Directorio Oficial de Establecimientos Año 2010 del Ministerio de Educación. Las unidades de análisis son los profesores que impartieron el curso de Historia, Geografía y Ciencias Sociales en primero y/o segundo año de enseñanza media durante los años 2010 y 2011, y los estudiantes que, habiendo asistido a las clases de esos profesores, se encuentren cursando tercer año de enseñanza media durante el año 2012.

Se utiliza una muestra no probabilística, aleatoria estratificada con afijación proporcional. La muestra es uni-etápica para los profesores y bi-etápica para los estudiantes: se seleccionan los establecimientos, se elige(n) al (los) profesor(es) que ha(n) impartido clases a un mismo curso, y luego se identifica a los estudiantes. La técnica de recolección de información es la encuesta. Se entrega un cuestionario auto-administrado a profesores y a estudiantes. Los instrumentos se aplicaron en 97 establecimientos educacionales: 28 municipales, 59 particulares subvencionados y 10 particulares pagados. Se aplicaron instrumentos a 111 profesores y a 2.360 estudiantes. Se contó con la autorización de todos los directores y el asentimiento de todos los estudiantes, pero sólo con el consentimiento de 1.205 padres y/o apoderados de los estudiantes.

Se realizó un análisis estadístico descriptivo de la información recolectada. Luego, se construyó una tipología de profesores según su estrategia de posicionamiento frente a la enseñanza de los 'temas controversiales'. Para ello se utilizaron algunas de las preguntas del cuestionario que fueron construidas para operar como indicadores de la estrategia de acción del profesor. Estos indicadores fueron establecidos en función de los constructos teóricos con que se realiza la investigación.

\section{RESULTADOS}

\subsection{CONFLICTIVIDAD DE LOS TEMAS Y SU ENSEÑANZA}

De los temas incluidos en los Programas de los cursos de Historia, Geografía y Ciencias Sociales de primero y segundo medio, se definieron quince 'temas controversiales' que 
Estudios Pedagógicos XLI, Nº 1: 275-292, 2015

ENSEÑANZA DE 'TEMAS CONTROVERSIALES' EN LA ASIGNATURA DE HISTORIA Y CIENCIAS SOCIALES

DESDE LA PERSPECTIVA DE LOS PROFESORES

fueron presentados a los profesores. De estos tópicos, según los profesores, son temas muy controversiales: 'Terrorismo de Estado y violación de los Derechos Humanos en América Latina' (65,14\%); 'Crecimiento económico y distribución de la riqueza' (52,38\%); 'La disminución y destrucción de la población indígena' (51,82\%); 'Los movimientos revolucionarios en América Latina' (51,43\%); 'El genocidio judío y las políticas de exterminio de pueblos' (48,60\%), y; 'La guerra fría' (40,37\%). Son relativamente controversiales: 'La conformación del territorio nacional' (37,84\%); 'El rol del Estado en el desarrollo social y económico' (37,61\%); 'Crisis del Estado de bienestar e implementación de políticas neoliberales' (36,7\%), y; 'Efectos de la globalización' $(37,04 \%)$. Son temas poco controversiales: 'El imperialismo y el reparto del mundo' (59,63\%); 'La inserción de Chile en la economía mundial en el siglo XIX' (49,54\%); 'Políticas públicas y privadas de control de la natalidad' (44,95\%); 'La Revolución Rusa' (47,17\%), y; 'La conquista española' $(45,05 \%)$ (Ver Gráfico $\left.\mathrm{N}^{\circ} 1\right)$.

Gráfico $N^{\circ} 1$. Nivel de conflictividad de los 'temas controversiales'

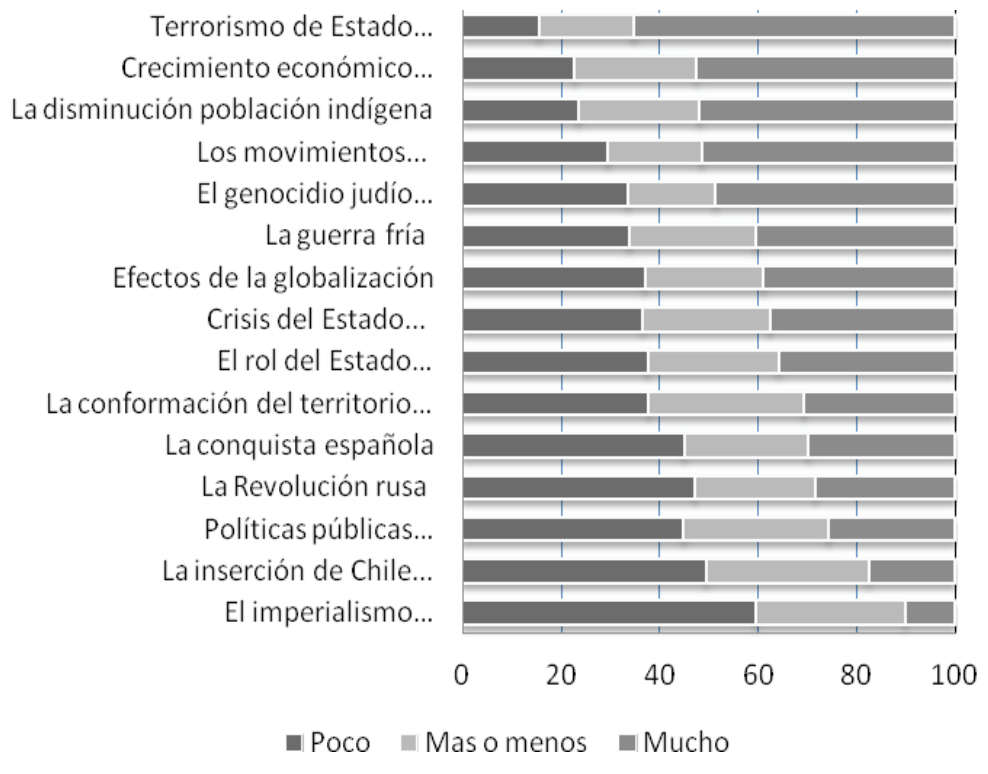

Fuente: elaboración propia.

Los 'temas controversiales' menos enseñados son: 'Terrorismo de Estado y violación de los Derechos Humanos en América Latina' (36,23\%), 'Crecimiento económico y distribución de la riqueza' $(25,00 \%)$ y 'Los movimientos revolucionarios en América Latina' (21,57\%). Los tópicos identificados como menos controversiales menos enseñados son: 'Crisis del Estado de bienestar e implementación de políticas neoliberales' $(28,21 \%)$, ‘El rol del Estado en el desarrollo social y económico' (21,05\%), y ‘La conformación del territorio nacional' $(18,18 \%)$. Y un tema muy poco controversial muy poco enseñado es: 'Políticas públicas y privadas de control de la natalidad' (37,04\%) (Ver Gráfico N²). 
Gráfico $N^{\circ} 2$. Enseñanza de los ‘temas controversiales’ según nivel de conflictividad

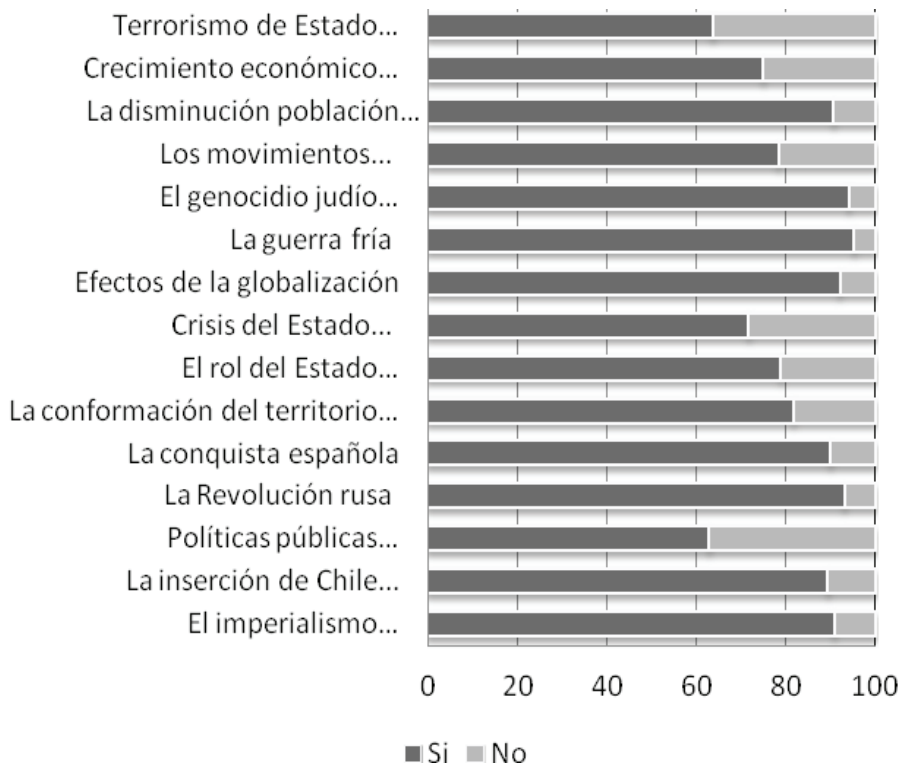

Fuente: elaboración propia.

\subsection{POSICIONAMIENTO FRENTE A LA ENSENAANZA DE 'TEMAS CONTROVERSIALES'}

Según las acciones que despliegan los profesores en la sala de clases, el 65,5\% es 'neutral', y el $22,1 \%$ combina conductas propias de un profesor 'objetivo' y de uno 'neutral'. De los profesores 'neutrales', el 46,0\% actúa como 'neutral activo', el 31,0\% como 'neutral comprometido' y el 6,2\% como 'crítico'. Aunque existen profesores 'evitativos puros', 'evitativos-neutrales', ‘evitativos-objetivos-neutrales', 'objetivos puros', ‘objetivosnegadores-neutrales' y 'neutrales-adoctrinadores', no son estadísticamente significativos. Ningún profesor se clasifica como 'adoctrinador' ni 'negador'.

El 25,66\% de los profesores dice enseñar sólo los aspectos objetivos ('objetivo'), pero al mismo tiempo, el 96,43\% declara presentar diferentes interpretaciones sobre un mismo acontecimiento ('neutral'). Además, un 37,17\% de los profesores reporta asumir la posición de los oprimidos ('crítico') cuando presenta un 'tema controversial', y el 7,8\% presenta su propia interpretación de los hechos, ya que considera que es la correcta ('adoctrinador').

\subsection{SOBRE LO QUE LOS PROFESORES ESPERAN DE LOS ESTUDIANTES}

Para el 99,12\% de los profesores es importante promover el diálogo y la discusión sobre los 'temas controversiales' enseñados ('neutral'), el 98,21\% declara que los estudiantes deben manifestar sus opiniones sobre los contenidos presentados por el profesor ('neutral'), y ningún profesor indica que los estudiantes no deben cuestionar ni criticar lo enseñado por el profesor ('adoctrinador por contenido'). El 12,39\% considera que el rol del estudiante es escuchar y aprender los contenidos que enseña el profesor ('adoctrinador por método'), y 
el 97,35\% piensa que los estudiantes deben reflexionar sobre los temas y sacar sus propias conclusiones ('neutral').

El 95,58\% de los profesores declara estar de acuerdo con que los estudiantes desarrollen una actitud crítica sobre la sociedad en que viven ('neutral'), mientras que el $7,14 \%$ espera que los estudiantes se adapten y respeten las normas y los valores sociales sin cuestionarlos. El 92,04\% de los profesores tiene la expectativa de que los estudiantes construyan su propia interpretación de la historia, mientras que para el $8,85 \%$ su meta es que los estudiantes memoricen los contenidos del curso.

\subsection{ROL DEL PROFESOR}

El 59,29\% de los profesores evita influir en las opiniones de los estudiantes ('neutral'); el $12,39 \%$ bromea, ironiza o se burla de ellos para mostrar que no está de acuerdo con algunas interpretaciones de la historia ('adoctrinador'); el 16,81\% apela a las emociones para convencer a los estudiantes sobre su interpretación de los hechos ('adoctrinador por método'); el 60,18\% de los profesores plantea sus opiniones aunque no concuerden con las ideas de los historiadores ('adoctrinador por contenido'); el 7,96\% reconoce que, aunque no tenga evidencias que sostengan sus argumentos, plantea sus ideas sobre la historia ('adoctrinador por contenido'); un 7,08\% considera que el profesor debe indicar la interpretación correcta de la historia ('adoctrinador por intensión'), y; el 3,54\% considera que los estudiantes no tienen conocimientos y/o capacidades suficientes para opinar sobre la historia ('adoctrinador por intención').

\subsection{SOBRE LA HISTORIA}

El 76,99\% de los profesores considera que hay personas que, por razones políticas o intereses personales, quieren distorsionar algunas partes de la historia ('adoctrinador por contenido'), y el 94,69\% indica que siempre es necesario presentar evidencias cuando uno expone su interpretación sobre los acontecimientos históricos ('neutral'). El 26,55\% considera que se puede seleccionar interesadamente las evidencias históricas para fundamentar lo que se desee ('adoctrinador por contenido'), para el 4,42\% lo importante son las ideas que se exponen y no los argumentos que las sustentan ('adoctrinador por contenido'), y el 69,91\% considera que se pueden tener ideas sobre la historia que no son compartidas por los historiadores ('adoctrinador por contenido'). No es significativo el número de profesores que piensan que la historia no debe ser criticada ('adoctrinador por contenido'), que hay sólo una interpretación verdadera de la historia ('negador'), y que no es necesario fundamentar las interpretaciones de la historia que no se comparten ('adoctrinador por contenido').

\subsection{COMPORTAMIENTO DE LOS ESTUDIANTES}

Cuando se presentan distintas interpretaciones sobre un mismo acontecimiento, según el $61,95 \%$ de los profesores, siempre o frecuentemente los estudiantes expresan sus opiniones personales, y según un $62,50 \%$ los estudiantes siempre o frecuentemente piden al profesor que exprese su opinión personal. El 23,01\% de los profesores indica que los estudiantes siempre o frecuentemente se confunden con la existencia de diversas interpretaciones sobre 
un mismo acontecimiento, pero según el 18,58\% eso rara vez o nunca sucede. Para el $49,56 \%$ de los profesores los estudiantes siempre o frecuentemente se motivan con los 'temas controversiales'. Según el 38,94\% rara vez o nunca se producen conflictos entre los estudiantes, pero según el 18,58\% esto sucede siempre o muy frecuentemente.

\subsection{SOBRE LA REALIZACIÓN DE LAS CLASES}

El material más utilizado para planificar las clases son las imágenes $(85,84 \%)$, los documentales $(76,11 \%)$, las fuentes escritas $(76,11 \%)$, las películas $(71,68 \%)$, el texto del profesor $(70,80 \%)$, el texto del estudiante $(70,80 \%)$, los textos especializados de historia $(62,83 \%)$ y textos generales $(61,95 \%)$.

La clase expositiva es la actividad más realizada (94,69\%). La siguen presentación de material audiovisual (88,50\%), búsqueda de información en Internet (76,99\%), construcción de cuadros, esquemas y/o marcos conceptuales (66,37\%), investigación $(65,49 \%)$, exposición $(63,73 \%)$, conversación sobre un tema $(61,06 \%)$, presentación de gráficos y/o esquemas $(59,29 \%)$, lectura de un texto $(59,29 \%)$, construcción de interpretaciones sobre acontecimientos $(56,64 \%)$, construcción de líneas de tiempo $(52,21 \%)$, búsqueda en libros, enciclopedias $(51,33 \%)$, escritura de ensayos $(49,56 \%)$, lectura dirigida $(47,79 \%)$, presentación de noticias de la época (46,90\%), debates (41,59\%), aplicación de cuestionario $(40,71 \%)$, testimonios $(38,94 \%)$, visitas a lugares de interés $(38,05 \%)$, escucha de música $(37,17 \%)$, frases para completar $(37,17 \%)$, realización de entrevistas a familiares $(32,74 \%)$, elaboración de noticieros o diarios de la época $(23,89 \%)$, lectura de libros sobre la época $(21,24 \%)$, elaboración de fichas $(13,27 \%)$, construcción de portafolios $(10,62 \%)$, copia de un texto $(7,08 \%)$ y memorización de un texto $(1,77 \%)$.

Los profesores declaran que, cuando enseñan un 'tema controversial', solicitan el análisis de documentos $(92,92 \%)$, construyen conceptos $(80,53 \%)$, analizan iconografías $(66,37 \%)$, organizan debates $(50,44 \%)$, solicitan ensayos $(48,67 \%)$, realizan talleres documentales $(42,48 \%)$, y realizan talleres bibliográficos $(21,24 \%)$. La estrategia didáctica privilegiada es la identificación de causas y consecuencias $(93,81 \%)$, seguida del análisis de fuentes históricas $(82,30 \%)$, aplicación de conceptos históricos $(75,22 \%)$, identificación de similitudes y diferencias entre procesos históricos y sociales $(73,45 \%)$, crítica de fuentes históricas, presentación de relatos sobre la historia $(48,67 \%)$, realización de trabajos de investigación $(47,79 \%)$, aplicación de categorías temporales $(40,71 \%)$, y formulación de hipótesis $(38,94 \%)$. Los materiales didácticos más utilizados son las imágenes $(87,61 \%)$, luego el texto del estudiante $(84,07 \%)$, los documentos históricos $(78,76 \%)$, los documentales $(77,88 \%)$, las películas $(71,68 \%)$, los textos de historiadores $(61,06 \%)$, los cuadros estadísticos $(53,98 \%)$, los diarios y revistas $(47,79 \%)$, los testimonios $(38,94 \%)$, y las salidas a terreno $(30,09 \%)$.

Para evaluar, los profesores utilizan preguntas de desarrollo (92,92\%), preguntas con respuestas de selección múltiple $(73,45 \%)$, exposición oral $(62,83 \%)$, elaboración de un texto $(60,18 \%)$, realización de una investigación $(54,87 \%)$, representación de roles $(19,47 \%)$, y resolución de problemas $(17,70 \%)$. Los profesores declaran que evalúan la actitud reflexiva y propositiva $(84,07 \%)$, la comprensión de la temporalidad y la historicidad $(83,19 \%)$, las habilidades comunicativas de carácter oral y escrito $(69,91 \%)$, el uso pertinente de conceptos de historia y ciencias sociales $(69,03 \%)$, la búsqueda y organización de información $(60,18 \%)$, los contenidos disciplinarios (54,87\%), la 
Estudios Pedagógicos XLI, N 1: 275-292, 2015

ENSENAANZA DE 'TEMAS CONTROVERSIALES' EN LA ASIGNATURA DE HISTORIA Y CIENCIAS SOCIALES

DESDE LA PERSPECTIVA DE LOS PROFESORES

comprensión del espacio (52,21\%), la comprensión de la empatía histórica $(48,67 \%)$, la ubicación espacial $(48,67 \%)$, y el conocimiento de fechas, personajes e hitos $(30,97 \%)$.

\section{DISCUSIÓN}

\subsection{GRADO DE CONTROVERSIALIDAD}

El grado de controversialidad otorgado por los profesores a los contenidos curriculares presentes en los Programas del Sector de Historia, Geografía y Ciencias Sociales del primer ciclo de Enseñanza Media, se asocia con la emocionalidad que ellos desatan cuando son analizados. Esta emocionalidad surge porque los tópicos impactan directamente sobre las interacciones que los sujetos establecen en su vida cotidiana y definen sus condiciones de existencia.

En el caso del golpe de Estado de 1973, el tópico es controversial no sólo porque la memoria colectiva de los chilenos que refiere al pasado reciente está fragmentada, sino también porque muchas de las víctimas más o menos directas de estos acontecimientos consideran que no han sido reconocidas como tales y/o que el sufrimiento no ha sido reparado, y porque quienes cometieron estas acciones, en muchos casos, permanecen impunes. Cuando se analiza el tema del crecimiento económico y la distribución de la riqueza, la emocionalidad emerge dado que en el país la desigualdad es extrema y continúa aumentando. Por tanto, muchos de los profesores y estudiantes viven directa o indirectamente las consecuencias de la desigualdad. Y cuando se trata el tema de la disminución y destrucción de la población indígena, hay que considerar que, al menos uno de diez estudiantes y algunos de los profesores, pertenecen a un pueblo originario y que el 'conflicto indígena' ha alcanzado estos últimos años grados extremos de violencia.

Considérese también que los temas identificados por los profesores como los más controversiales aluden a los derechos fundamentales de las personas: derechos humanos, derechos económicos y derechos de los pueblos originarios. Son temas que impactan a todos, porque están vinculados con el derecho a la vida. Ellos han sido reivindicados por diferentes movimientos sociales y aún lo siguen haciendo. Pese a esto, y aunque existen políticas públicas destinadas a atenderlos y directrices internacionales para abordarlos, estas problemáticas aún no han sido resueltas.

De lo anterior se evidencia que la concepción anglosajona de 'temas controversiales' antes expuesta, no considera la dimensión de la actualidad en la sociedad que debate el tópico, lo que ha sido bien explicitado cuando se acuñó el término francés 'cuestiones socialmente vivas'. Este concepto enfatiza que estos tópicos no están resueltos ni por la sociedad ni por la ciencia, que desatan emociones, movilizan valores e intereses, son políticamente sensibles, impelen a la toma de posición y agregan la dimensión de la incertidumbre (Simonneaux, 2011; Tutiaux-Guillon, 2010; Legardez et Simonneaux, 2006). Además, resulta necesario remarcar el carácter socio-histórico de la controversialidad que Cusack (2009) ya había señalado.

\subsection{LO QUE SE ENSEÑA Y NO SE ENSEÑA}

La fuerza con que estos 'temas controversiales' cruzan la vida de los profesores, podría explicar la posición 'evitativa' que asumen algunos de ellos cuando deben enseñarlos, es 
decir, reconocen que se trata de un 'tema controversial' pero no lo trabajan en clases. Evitan enseñar los tópicos polémicos que están directamente vinculados con la acción del Estado.

Esto da cuenta del temor a cuestionar las estructuras sociales vigentes, lo que probablemente tiene su origen en que son parte de una sociedad que -como lo indica Lechner (2002)- teme al conflicto. Además, son miembros de una sociedad que aún no ha superado el temor que se internalizó durante la dictadura (1973-1990) cuando se utilizó la amenaza y el miedo como recurso para controlar la acción política. En ese tiempo, el temor se trasladó a la vida cotidiana y se hizo crónico (Lira y Castillo, 1991). Entonces, pareciera que este miedo aún no ha sido del todo superado debido a que su instalación transformó las relaciones sociales, y se ha reproducido porque las relaciones sociales no se han modificado. Así, sucede que el miedo a la autoridad del Estado se reproduce en los espacios locales, con la autoridad municipal, los sostenedores y los propietarios. Luego se traspasa hacia los padres, un actor de la comunidad escolar recientemente empoderado, y que puede intervenir cuando en la escuela se cuestiona lo transmitido en las familias.

En este sentido, el profesor prefiere no abordar los temas que resultan altamente controversiales. Asumen una posición 'evitativa', pero no se reconocen a sí mismos en esa postura, aunque no están cubriendo la totalidad de los contenidos que mandata enseñar el currículum oficial nacional. De este modo, silencian sus críticas al modelo neoliberal y son cautelosos de no aparecer ante los demás como introduciendo la política en la escuela. A esto se suma el temor al desborde de la clase cuando los estudiantes manifiesten sus opiniones sobre la sociedad actual. Se trata del miedo al descontrol que Lechner (2002) describe entre los miedos históricos de la sociedad chilena, como una expresión del miedo al conflicto.

Por el contrario, los tópicos más enseñados por los profesores son aquellos que se consideran como menos controversiales, salvo el que refiere a las 'Políticas públicas y privadas de control de la natalidad', porque se entrecruza con la formación religiosa que los estudiantes reciben en la familia y/o en la misma escuela. Entonces, sí se enseña lo que no es controversial y no se enseña lo que sí lo es. Lo que se evita es el conflicto. Se evidencia que los profesores soslayan el conflicto que, al enseñar los 'temas controversiales', podrían generarse con autoridades educacionales, apoderados y/o estudiantes.

\subsection{POSICIONAMIENTO ANTE LOS 'TEMAS CONTROVERSIALES'}

Según lo declarado, los profesores de Historia, Geografía y Ciencias Sociales no conocen a nivel teórico las distintas posiciones que un docente puede asumir ante la enseñanza de un 'tema controversial', puesto que éste no es un tema que haya sido incluido en su formación inicial en las instituciones universitarias ni durante su formación continua. Además, se evidencia una distancia entre el nivel declarativo de las prácticas y las acciones realizadas, es decir, los profesores declaran asumir una posición -frecuentemente la 'neutralidad'-, mientras que sus acciones no se corresponden con ese posicionamiento. Más bien, sucede que combinan acciones propias de diferentes posiciones, que pueden ser hasta contradictorias o se anulan entre sí, dado que la tipología de estrategias de posicionamiento para la enseñanza de los 'temas controversiales' expuesta es excluyente, es decir, no permite ser parte de dos o más categorías al mismo tiempo. Esto da cuenta de la carencia de un modelo pedagógico específico que permita abordar los 'temas socialmente vivos' en la sala de clases. 
Estudios Pedagógicos XLI, Nº 1: 275-292, 2015

ENSEÑANZA DE 'TEMAS CONTROVERSIALES' EN LA ASIGNATURA DE HISTORIA Y CIENCIAS SOCIALES

DESDE LA PERSPECTIVA DE LOS PROFESORES

Cuando los profesores declaran su posicionamiento ante la enseñanza de los 'temas controversiales', aunque son pocos, algunos de ellos no consideran los tópicos polémicos como parte de los contenidos que deben trabajar en su curso; los eliminan de su acción pedagógica debido al temor de entrar en un conflicto. Adicionalmente, uno de cada cuatro profesores declara posicionarse ante los 'temas controversiales' como 'objetivo', lo que significa que ellos sólo enseñarían en sus clases 'lo objetivado', aquello que no produce discusión. Esto no se alinea con el saber disciplinario actualizado, a saber, que la historia es un relato que da cuenta de una interpretación del pasado. Según este postulado, no hay posibilidad alguna de identificar un fragmento del contenido curricular del curso de historia que sea objetivo.

Positivamente, la mayoría de los profesores se identifica como 'neutrales-activos', es decir, declaran presentar diversas interpretaciones sobre un mismo acontecimiento, pero evitan manifestar su opinión sobre lo expuesto. Sólo un número reducido de ellos explicita su interpretación de los acontecimientos frente a los estudiantes, es decir, se declara 'neutral-comprometido'. Esta situación se relaciona directamente con lo planteado sobre los temores que tienen los profesores de manifestar sus opiniones, tanto frente a las autoridades como frente a los estudiantes.

Cabe considerar que, efectivamente, no todos los profesores están acogidos al Estatuto Docente. Por ello, aunque efectivamente manifestaran una opinión ideológica y/o política, podrían iniciar un conflicto con el contratante de su labor pedagógica, lo que, a su vez, podría poner en riesgo su puesto de trabajo que depende de un sostenedor o de un propietario del establecimiento. Lo que devela esta situación es la pervivencia de la representación social de la escuela como un espacio donde ni la política ni el conflicto tienen lugar. Según esto, en la escuela sólo tendría cabida el saber consensuado y 'objetivado'. Entonces, muchos profesores actúan de acuerdo a la representación social que indica que la escuela debe mantenerse separada del acontecer social.

Además, en la posición 'neutral activa' asumida mayoritariamente por los profesores, subyace la idea de que la educación es una acción libre de juicios morales, siendo que la educación no puede ser una acción neutral, y que los profesores tienen la obligación de orientar valóricamente a los estudiantes. En términos disciplinarios, cuando se enseña historia, los profesores -a la luz de los avances disciplinarios-, deberían asumir que los relatos históricos, como ya se dijo, no son objetivos sino que corresponden a interpretaciones del pasado. Por tanto, el profesor necesariamente debería seleccionar uno o varios de esos relatos, e indicar algunos antecedentes de quienes los han construido y/o asumido, así como del lugar social donde ellos se encuentran. Más aún, cuando el profesor enseña 'cuestiones socialmente vivas' en la sociedad de la cual es miembro, él mismo tiene una posición sobre el tópico. Entonces, no puede ocultar su posición a los estudiantes. Si lo hace, modela una postura de no compromiso con el acontecer social, lo que no se corresponde con las exigencias de la escuela de formar ciudadanos activos, democráticos y deliberantes.

\subsection{SOBRE LA FORMACIÓN INICIAL DE LOS DOCENTES}

Sucede que la noción de 'tema controversial' generalmente no está incluida en la formación inicial de los profesores de historia, ni tampoco se suelen cubrir los tópicos que generan polémicas, porque se trata de 'cuestiones socialmente vivas' que están más próximas a la sociología y a la ciencia política, que a lo que se concibe generalmente como parte del 
dominio histórico. Además, los profesores fueron formados, tanto en su educción escolar como universitaria, bajo prácticas tradicionales de enseñanza, con una concepción a su vez tradicional de la historia y de las estrategias pedagógicas que les eran propias: clases expositivas, énfasis en la descripción detallada de los hechos, memorización, restitución del saber del profesor. Por consiguiente, reproducen su propia experiencia pedagógica, lo que reduce la posibilidad de mirar el pasado y la acción pedagógica desde una perspectiva crítica.

Por la misma situación, entre los actuales profesores pervive la concepción de la historia como un relato sobre el pasado, al cual se debe acceder desde una perspectiva cronológica. Esto determina que, si pone en práctica la lógica lineal para enseñar la historia, no se alcanzan a cubrir los temas que están más próximos al presente, que son precisamente los tópicos que producen polémicas a nivel social. Además, la mantención de una concepción lineal, cronológica y secuencial de la historia, impide cubrir el currículum de una manera más integrada y vinculada a las ciencias sociales, es decir, enseñar historia a partir de las temáticas que más motivan a los estudiantes.

\subsection{DISCURSO Y ACCIÓN}

Declarar asumir la posición 'neutral' cuando se enseña un 'tema controversial' es responder según lo esperado socialmente, y también en coherencia con los modelos pedagógicos promovidos, tanto por las ciencias sociales como por las autoridades del Ministerio de Educación. No obstante, esta declaración de 'neutralidad' se contradice con la declaración de las acciones realizadas en la sala de clases cuando se enseñan las 'cuestiones socialmente vivas'.

Las prácticas declaradas por los profesores evidencian la pervivencia de acciones educativas tradicionales, a saber: clases expositivas, donde predomina el análisis de documentos, de identificación de causas y consecuencias de los hechos sociales como estrategia didáctica, donde se utiliza la imagen como material didáctico y las preguntas de desarrollo como recurso para realizar las evaluaciones. También se constata una distancia entre la expectativa que tienen los profesores sobre los estudiantes y la relación pedagógica que se establece con ello: en el discurso se valora su participación en la sala de clases, pero las actividades, estrategias y procedimientos que despliegan los profesores para cubrir los contenidos curriculares no lo permiten, ya que prima la exposición del discurso del profesor desde un lugar de saber-poder sobre lo acontecido en la historia, la memorización, la evaluación concebida como una instancia de restitución del saber del profesor y el rol pasivo del estudiante. Hay pocas ocasiones en donde los estudiantes realicen investigaciones o se organicen debates que conduzcan a la construcción del saber histórico. Muy ocasionalmente se planifican acciones que permitan la construcción de interpretaciones, que promuevan un diálogo fundado en argumentos, y que favorezcan el desarrollo del pensamiento crítico. No hay espacio para el conflicto. Las acciones llevadas a cabo no apuntan a lograr la expectativa declarada por los profesores sobre los estudiantes, a saber: que éstos desarrollen una actitud crítica sobre la realidad en que viven.

Esta diferencia que se constata entre el discurso y la acción puede tener su origen en carencias específicas para enfrentar la enseñanza de los 'temas controversiales', puesto que esto exige a los profesores conocimientos especializados sobre tales temas, es decir, requieren de conocimientos no sólo de la historia como disciplina, sino también de las 
otras ciencias sociales que no siempre han sido consideradas con amplitud en la formación inicial docente. Al mismo tiempo, los profesores requieren del desarrollo de competencias para la gestión del grupo, y de metodologías específicas que promuevan la participación de los estudiantes concebidos como sujetos capaces de expresar opiniones.

La distancia que se evidencia entre el discurso y la acción también puede deberse a que los profesores reproducen modelos pedagógicos tradicionales sin incorporar la reflexión sobre su práctica. Crandall et al. señalan: "los maestros tienden a actuar por intuición y rara vez dedican tiempo a razonar acerca de cómo realizan su tarea" (1982: 29 Cit. en Fullan y Stiegelbauer, 2009: 39). Si los profesores reflexionaran sobre su práctica pedagógica tensionarían la relación profesor-estudiante, cuestionarían el lugar de podersaber que detenta el profesor bajo el modelo tradicional de educación. Comprenderían que asumir la 'neutralidad' inevitablemente distribuye el poder entre los participantes de la acción pedagógica, es decir, el poder ya no se ubicaría en un rol sino en la capacidad argumentativa. Si los argumentos son razonables, son válidos indistintamente de quien provengan, del profesor o de los estudiantes. Esto implica concebir una nueva organización de la sala de clases que resulte adecuada para la implementación de metodologías participativas y dialógicas, que se contraponen con las relaciones de poder que son propias de los establecimientos escolares. Esto podría desembocar en una transformación de la institución o en un conflicto con las autoridades institucionales tradicionales que se resisten a los procesos de cambio.

La distancia entre discurso y acción pedagógica cuando se enseñan 'temas controversiales' también puede estar relacionada con el tópico trabajado y con el posicionamiento del profesor como sujeto ante el conflicto social. Si el profesor está muy involucrado con el 'tema controversial', exponer ciertas interpretaciones de los acontecimientos puede generarle inseguridades y temores. Entonces, como mecanismo psicológico de defensa, podría evitar enseñar algunos de los contenidos que lo implican en demasía. Por tanto, evita asumir la 'neutralidad' y/o evita manifestar su opinión personal. Así, el profesor se libera del conflicto que le produce consigo mismo y con los otros el hecho de tratar las 'cuestiones socialmente vivas'.

Esta diferencia que se manifiesta cuando se enseñan 'temas controversiales' en el curso de historia puede ser también una manifestación de un fenómeno mayor, vinculado particularmente con la instalación de la Reforma Curricular de 1996. Ésta promovió la puesta en práctica de estrategias pedagógicas crítico-dialógicas y, en consecuencia, se organizaron numerosas instancias de capacitación, pero las transformaciones no han impactado directamente en la sala de clases como se ha observado en investigaciones previas (Toledo, Magendzo \& Gazmuri, 2011). Esto puede interpretarse como una acción de resistencia. Se trataría de una resistencia al cambio debido al alto nivel de exigencia al que están sometidos los profesores, y las deficitarias condiciones en que desarrollan sus actividades laborales (Fullan y Stiegelbauuer, 2009). Entonces, los profesores resistirían el cambio de prácticas en el aula en todas las disciplinas escolares, entre ellos los profesores de Historia, Geografía y Ciencias Sociales. A pesar de ello, no se observan manifestaciones explícitas de cuestionamiento al cambio.

Finalmente, también se puede agregar que esta distancia entre el discurso y la práctica responde a que nuestra sociedad aún debe ser considerada desde la pedagogía como una sociedad post-conflicto (Geschier, 2010; Ferreira \& Janks, 2009; Keet, Zinn \& Porteus, 2009), una sociedad que aún no modifica las relaciones dañadas a causa de las violaciones 
a los derechos humanos. Por eso se evita tensionar, disentir, debatir, dialogar y manifestar opiniones críticas al sistema social. En este sentido, es de mayor urgencia incorporar la conflictividad en la escuela, dado que ése es el lugar privilegiado para que las nuevas generaciones puedan aprender a argumentar, disentir, dialogar y tomar posiciones. Sólo así los actuales estudiantes podrán constituirse en ciudadanos participativos y ser activos en la construcción de una sociedad más democrática, diversa y tolerante.

\section{CONCLUSIONES}

Los temas que los profesores identifican como más controversiales tienen relación directa con la violación a los derechos fundamentales de las personas. Estos temas más polémicos se enseñan menos que los que son identificados con un menor grado de controversialidad, lo que evidencia que los profesores evitan el conflicto.

Aunque los profesores no han sido formados para enseñar los temas que dividen a la sociedad, se auto-posicionan como 'neutrales', es decir, declaran exponer distintas interpretaciones sobre un mismo hecho o situación. Específicamente, se definen como 'neutrales-activos', es decir, evitan manifestar su posición sobre los tópicos tratados, lo que evidencia su temor a manifestar sus posiciones críticas ante el ordenamiento social. Sólo una pequeña parte de los profesores se identifica como 'neutral-comprometido', es decir, manifiesta públicamente su opinión sobre los acontecimientos que enseña. Sin embargo, sus prácticas 'neutrales' no se corresponden con las acciones propias de esta postura. Las acciones desplegadas en la sala de clases se asocian con el modelo tradicional de enseñanza de la historia. Entonces, se devela una distancia entre el discurso y la acción en relación a la enseñanza de estos tópicos en disputa.

\section{REFERENCIAS BIBLIOGRÁFICAS}

Advisory Group on Citizenship (1998). Education for Citizenship and the Teaching of Democracy in Schools. London: Qualifications and Curriculum Authority.

Apple, M. (2008). Ideología y currículo. Madrid: Ediciones Akal.

Asimeng-Boahene, L. (2007). Creating Strategies to deal with Problems of Teaching Controversial Issues in Social Studies in African Schools. Intercultural Education, vol.18 (3), 231242.

Barton, K. \& Mc Cully, A. (2007). Teaching Controversial Issues... where Controversial Issues Really Matter. Teaching History, (127), 13-19.

Bridges, D. (1979). Education, Democracy and Discussion. Windsor: NFER.

Byford, J., Lennon, S. \& Russell, W. (2009). Teaching Controversial Issues in the Social Studies: A Research Study of High School Teachers. The Clearing House of Social Studies, vol.82 (4), 165170.

Carr, P. (2011). En busca de la democracia... a través de la pedagogía crítica. Postconvencionales, (3), 1-3.

Clarke, P. (2005). Teaching Controversial Issues: A Four-step Classroom Strategy for Clear Thinking on Controversial Issues. Retrieved on October 3, 2010 from http://bctf.ca/globaled/ teachingresources/clarkepat/teachingcontroversialissues.html

Corngold, J. \& Waddington, D. (2006). Teacher Neutrality Reconsidered: Implications for the 
Estudios Pedagógicos XLI, № 1: 275-292, 2015

ENSEÑANZA DE 'TEMAS CONTROVERSIALES' EN LA ASIGNATURA DE HISTORIA Y CIENCIAS SOCIALES

DESDE LA PERSPECTIVA DE LOS PROFESORES

Political Science Classroom. Annual Meeting of American Political Science Association. Philadelphia, United States.

Cotton, D. (2006). Teaching Controversial Environmental Issues: Neutrality and Balance in the Reality of the Classroom. Educational Research, vol.48 (2), 223-241.

Cross, R. \& Price, R. (1996). Science Teacher's Social Conscience and the Role of Teaching Controversial Issues in the Teaching of Science. Journal of Research in Science Teaching, vol.33 (3), 319-333.

Cusack, M. (2009). Policing Matters: Addressing the Controversial Issue of Policing through

Education for Reconciliation. International Review of Education, vol.55 (2-3), 251-267.

Cuypers, S. \& Ishtiyaque, H. (2006). Education for Critical Thinking, Can it be non Indoctrinative?

Educational Philosophy and Theory, vol.38 (6), 724-727.

Dawson, V. (2001). Addressing Controversial Issues in Secondary School Science. Australian

Science Teachers Journal, vol.47 (4), 38-44.

Ezzedeen, S. (2008). Facilitating Class Discussions around Current and Controversial Issues.

Ten Recommendations for Teachers. College Teaching, vol.56 (4), 230-236.

Ferreira, A. \& Janks, H. (2009). Doves, Rainbows and an Uneasy Peace: Student Images of

Reconciliation in a Post-conflict Society. Perspectives in Education, vol.27 (2), 134-135.

Finkin, M., Post, R., Nelson, C., Benjamin, E. \& Combest, E. (2007). Freedom in the Classroom.

New Directions for Student Services, vol.7 (59), 54-61.

Finn, G. (1990). Children and Controversial Issues: Some Myths and Misinterpretations and Challenged from a Cognitive-developmental Perspective. Cambridge Journal of Education, vol.20 (1), 5-24

Fullan, M. y Stieegelbauer, S. (2009). El cambio educativo. Guía de planeación para maestros. México D.F.: Editorial Trillas.

Gayford, C. (2002). Controversial Environmental Issues: A Case Study for the Professional Development of Science Teachers. International Journal of Science Education, vol.24 (11), 11911200.

Gazmuri, C. (s/f). Una interpretación política de la experiencia autoritaria (1973-1990).

Recuperado el 15 de abril desde http://www.archivochile.com/Dictadura_militar/doc_sobre_dm/

DMdocsobre0007.pdf

Gerson, M. (1997). Teaching Democracy by Doing It. Educational Leadership, vol.54 (5), 6-11.

Geschier, S. (2010). Vulnerability and Belonging in the History Classroom: A Teacher's positioning in 'Volatile Conversations' on Racism and Xenophobia. Perspectives in Education, vol.28 (3), 23-33.

Hanks, C. (2008). Indoctrination and the Spaces of Reasons. Educational Theory, vol.58 (2), 193-194.

Harwood, A. \& Hahn, C. (1990). Controversial Issues in the Classroom. Retrieved on November 3, 2010 from www.eric.ed.gov

Harwood, D. (2001). The Teacher's Role in Democratic Pedagogies in UK Primary and Secondary Schools: A Review of Ideas and Research. Research Papers in Education, vol.16 (3), 293-319.

Hess, D. (2004). Controversies about Issues in Controversial Democratic Education. Political Science \& Politics, vol.37 (2), 257-261.

Hocutt, M. (2005). Indoctrination vs Education. Academic Questions, vol.18 (3), 35-43.

Huneeus, C. (2003). Chile: Un país dividido. La actualidad del pasado. Santiago: Catalonia.

Jacobs, G. (2010). The Academic Controversy Technique: Towards Cooperative Debates. Intercultural Education, vol.21 (3), 291-296.

Keet, A., Zinn, D. \& Porteus, K. (2009). Mutual Vulnerability: A Key Principle in a Humanising Pedagogy in Post-conflict Societies. Perspectives in Education, vol.27 (2), 109-119.

Lechner, N. (2002). Las sombras del mañana. La dimensión subjetiva de la política. Santiago: 
LOM.

Legardez, A. et Simonneaux, L. (2006). L'école à l'épreuve de l'actualité. Enseigner les questions vives. Paris: ESF Éditeur.

Levinson, R. (2006). Towards a Theoretical Framework for Teaching Socio-scientific Controversial Issues. International Journal of Science Education, vol.28 (10), 1201-1204.

Levitt, G. \& Longstreet, W. (1993). Controversy and the Teaching of Authentic Civic Values. The Social Studies, vol.84 (4), 142-47.

Lira, E. y Castillo, M. I. (1991). Psicología de la amenaza política y del miedo. Santiago: Instituto Latinoamericano de Salud Mental y Derechos Humanos.

Merry, M. (2005). Indoctrination, Moral Instruction, and Non-rational Beliefs: A Place for Autonomy? Educational Theory, vol.55 (4), 405-409.

Moulian, T. (2010). El deseo de otro Chile. Santiago: LOM.

Osorio, J. (2007). Ocho ideas sobre formación ciudadana y escuela para la democracia: Un planteamiento post Movilización Pingüina. Paulo Freire. Revista de Pedagogía Crítica, vol.6 (4), 41-45.

Oulton, C., Day, V., Dillon, J. \& Grace, M. (2004). Controversial Issues-teachers Attitudes and Practices in the Context of Citizenship Education. Oxford Review of Education, vol.30 (4), 490-491.

Simonneaux, J. (2011). Les configurations didactiques des questions socialement vives économiques et sociales. Récupérée le 15 Juin à partir de http://oatao.univ-toulouse.fr/5289/1/ Simonneaux_5289.pdf

Sims, R. (2004). Business Ethics Teaching: Using Conversational Learning to Build an Effective Classroom Environment. Journal of Business Ethics, vol.49 (2), 201-211.

Stern, S. (2000). De la memoria suelta a la memoria emblemática: Hacia el recordar y el olvidar como proceso histórico. En M. Garcés (Ed.), Memoria para un nuevo siglo: Chile, miradas a la segunda mitad del siglo XX (pp. 11-33). Santiago: LOM.

Toledo, M. I., Magendzo, A. \& Gazmuri, R. (2011). Recent History Teaching in Countries where Human Right Violations have been experienced: Case Studies from Chile. Perspectives in Education, vol.29 (2), 19-27.

. (2010). La enseñanza de la historia del presente que da cuenta de las violaciones a los derechos humanos en la educación secundaria, según los profesores. En VVAA, Pedagogía de la memoria. Desafío para la educación en derechos humanos (pp. 126-140). Santiago: Heinrich Böll Stiftung Cono Sur.

y Magendzo, A. (2009). La enseñanza de la Sub Unidad "Régimen Militar y Transición a la Democracia" en Segundo Año de Enseñanza Media desde la perspectiva de los estudiantes. Revista Praxis, (15), 17-28.

(2007). Estudio cuantitativo y cualitativo de la enseñanza de la historia del presente de acuerdo al currículum de la Reforma, según los profesores, estudiantes y padres de $6^{\circ}$ básico: Identificación de buenas prácticas y metodologías de enseñanza. Informe de avance Proyecto FONDECYT $N^{\circ}$ 1085228. Santiago: Universidad Diego Portales.

., Veneros, D. y Magendzo, A. (2006). Lugares de la memoria: Activación de la memoria colectiva a través de la visita de estudiantes al Parque Por la Paz Villa Grimaldi. Informe Final Proyecto VRA-UDP. Santiago: Universidad Diego Portales.

Trilla, J. (1995). Educación y valores controvertidos. Elementos para un planteamiento normativo sobre la neutralidad en las instituciones educativas. Revista Iberoamericana de Educación, (7), 93120.

Tutiaux-Guillon, N. (2010). Les questions socialement vives en histoire et en géographie: Quelles analyses didactiques? VII Jornadas Internacionals de Recerca en Didàctica de les Ciències Socials. Barcelona, España.

Van Rooy, M. (2000). Controversial Issues within Biology Teaching. Australian Science Teachers Journal, vol.46 (1), 20-26. 
Estudios Pedagógicos XLI, N 1: 275-292, 2015

ENSEÑANZA DE 'TEMAS CONTROVERSIALES' EN LA ASIGNATURA DE HISTORIA Y CIENCIAS SOCIALES DESDE LA PERSPECTIVA DE LOS PROFESORES

Vandenberg, D. (2009). Critical Thinking about Truth in Teaching: The Epistemic Ethos. Educational Philosophy and Theory, vol.41 (2), 155-165.

Williams, R. (2006). Academic Freedom in Higher Education within a Conservative Sociopolitical Culture. Innovative Higher Education, vol.31 (1), 5-25. 